\title{
COVID-19: The Present Psychological Health and its Possible Outburst as Socio-economic Destabilization
}

\section{Debashish Ghose ${ }^{1 *}$, Suryakanta Swain ${ }^{2}$, Bikash Ranjan Jena ${ }^{3}$, Sanjeeb Kumar Sahoo ${ }^{4}$}

${ }^{1}$ Department of Pharmaceutics, Roland Institute of Pharmaceutical Sciences, Berhampur, Biju Patnaik University of Technology, India

${ }^{2}$ Department of Pharmaceutical Science, The Assam Kaziranga University, School of Health Sciences, India

${ }^{3}$ School of Pharmacy and Life Science, Centurion University of Technology and

Management, India

${ }^{4}$ Department of Pharmacology, Roland Institute of Pharmaceutical Sciences, Berhampur, Biju Patnaik University of Technology, India

*Corresponding Author: Debashish Ghose, Department of Pharmaceutics, Roland Institute of Pharmaceutical Sciences, Berhampur, Biju Patnaik University of Technology, India.
Received: March 29, 2021;

Published: May 20, 2021

(C) All rights are reserved by Debashish Ghose., et al.

\begin{abstract}
The current Covid-19 produced vital psychological and social effects on the population. Research has highlighted the impact on the most exposed groups' psychological well-being, including kids, university students, and health officials; they are further likely to produce post-traumatic anxiety dysfunction, stress, distress, and other signs of anxiety. The social way and the safeguard actions have affected people's association and their understanding of compassion toward others. From this prospect, telepsychology and technological devices assume important roles in decreasing the pandemic's negative effects. These tools present benefits that could improve patients' psychological treatment online, such as the possibility to meet from home or the workplace, saving money and time, and maintaining the relationship between therapists and patients.
\end{abstract}

Keywords: Covid-19; Telepsychology; Post-traumatic Anxiety

\section{Introduction}

With the advent of an unalarmed catastrophic coronavirus (COVID-19) outbreak, dictated by proliferative acute respiratory syndrome (SARS-CoV-2) infection in Wuhan's Chinese city, an extreme scenario of socio-economic imbalance and situational crisis with instinctive psychological distress struck worldwide [1,2]. Although cessation of social activities was put to a standstill in major continents and countries, all essential mobility and movements were barred due to lockdown and quarantine guidelines. Simultaneous- ly, the local hospitals worldwide suddenly started facing large proportions of new infections with the COVID-19 virus, which made them implement progressive strategies as per their emergency protocols [3].

With this condition of unpreparedness and sufficient knowledge regarding such alien viruses, the general population and the majority of the healthcare professionals became exposed to the sudden emotional impact of COVID-19 infection due to both the pandemic 
and its consequences worldwide. The catastrophe led to prolonged exposure to socio-emotional and physical stress [4]. Consequently, the worldwide domain of researchers boosted their efforts in knowing the socio-emotional levels of humans during this bizarre psychological condition. This proposed concern would be an excellent and effective guard-wall in mitigating the situational outcomes in the other possible future epidemics and pandemics. The social implications that are to be implemented require proper security protocol ineffective pandemic management can have varying degrees of outburst within the individuals [5]. Some population segments have shown their involvement in unwanted anxiety, feelings of depressive thoughts, and addiction to post-traumatic symptoms because of their excessive stress level during this pandemic.

Psychological stability and behavioral impact due quarantine and lockdown

The modern world in which all individuals can rapidly travel and communicate has been rarely forced to the current social isolation and restrictions, which correlates to the sense of frustration and uncertainty. This unprecedented situation related to the COVID-19 outbreak demonstrates that individuals are essentially and emotionally unprepared for the detrimental effects of biological disasters that directly show how everyone may be frail and helpless [6]. Social distancing and significant lockdown restrictions have been imposed in China and later in most European countries where Italy and Spain experienced a tragic growth of positive cases. However, most counties and their governmental regulations were essential to effectively maintain social equilibrium and witnessing the individuals-'individuals' safety, a direct only strategy that aimed to curb socio-economic issues raised due to COVID-19. Behavior-based experimental research reported that maximum individual subjects delivered with abrupt manifestations of psychological symptoms like; sudden emotional upsets (sense of suicidal tendency), depressional mood swings, mood alterations and consistent irritability, prolonged insomnia, post-traumatic stress symptoms, needless anger, and rapid emotional exhaustion were common among those who have been quarantined [7]. Long-term behavioral shifts like vigilant handwashing with sanitizers excessively and avoidance of large crowds and a delayed return to normality are most common even after many months after the quarantine was also reported. Hence, this imposed period of quarantine had a potentiated effect on the individual's mental health's altered psychological response .
Predictive psychological status and conditions

Uncleared fear of death due to infection

This condition is one of the most frequent possible psychological responses to any situational pandemics. Various studies and research showed individuals (s) with a higher risk of infection might develop a puzzling attitude of fears about their own and one's health, worries about infecting others, and fear of infecting family members. Jeong and his group reported that these categories of individuals are more vulnerable than others with actively prone to having the infection even several months after the exposure [8]. Various other studies listed that pregnant and younger adults are on the verge of more fear of becoming infected or transmitting the virus.

\section{Pervasive uneasiness}

Human retreat associated with restrictions, including lockdown steps, is linked to perceptions of ambiguity for the future, the anxiety of new and strange infective factors occurring in abnormally developed anxiety. Pressure may be directly related to sensorial loss and pervasive isolation, in this case, the first sleeplessness, but later distress and post-traumatic tension occurred. In extension, anxiety is closely linked with exhaustion and diminished representation in healthcare workers, while dullness and isolation are directly related to violence, disappointment, and difficulties related to quarantine restrictions. Moreover, supplementary destructive effects connected with pervasive tension in a pandemic time may include some noticed economic human assistance, detachment of loved individuals, end of independence, ambiguity, and apathy [9].

\section{Self-frustration and instinctive-stressed boredom states}

Circumstances like discomfort, boredom, social withdrawal, and defeat are directly related to custody, abnormally decreased human/environmental contact with others, and loss of normal practices. Jeong., et al. failure, and pervasive isolation appear to arise from the interference of regular liveliness, suspension of social obligations, not accepting part within social networking ventures [10]. They are predestinated, in this context, hopelessness, coincidentally with other individual characteristics such as the sense of youth abuse as considerably while advanced neural processing models may significantly and separately foretell self-destructive response. Still, even the unacceptable annoyance related to the imposition of quarantine may direct toward adverse outcomes. 


\section{Conclusion}

The adaptive community-based approaches to promote resilience and psychologically exposed individuals while the COVID-19 trauma is significant for each community. The psychological impact of worry and anxiety propelled by the sudden expanse of that pandemic need's attention as a public well-being priority for both authorities and policymakers who should rapidly comply with the distinct behavioral approaches to decrease the virus's strain and the dramatic psychic well-being outcomes of this fury.

\section{Bibliography}

1. Xiang YT., et al. "Timely mental healthcare for the 2019 novel Coronavirus outbreak is urgently needed". Lancet Psychiatry 7 (2020): 228-229.

2. Thakur V and Jain A. "COVID 2019-suicides: a global psychological pandemic”. Brain, Behavior, and Immunity 951 (2020): 952-953.

3. Zhai Y and Du X. "Loss and grief amidst COVID-19: a path to adaptation and resilience". Brain, Behavior, and Immunity 87 (2020): 80-81.

4. Duan L and Zhu G. "Psychological interventions for people affected by the COVID-19 epidemic". Lancet Psychiatry 7 (2020): 300-302.

5. Maunder R., et al. "The immediate psychological and occupational impact of the 2003 SARS outbreak in a teaching hospital". CMAJ 168 (2003): 1245-1251.

6. Barbisch D., et al. "Is there a case for quarantine? Perspectives from to Ebola". Disaster Medicine and Public Health Preparedness 9 (2015): 547-553.

7. Rubin GJ and Wessely S. "Coronavirus: the psychological effects of quarantining a city". BMJ Opinion 368 (2020): m313.

8. Sa L. "How much "Thinking" about COVID-19 is clinically dysfunctional". Brain, Behavior, and Immunity 87 (2020): 97-98.

9. https://covid19.who.int/
10. Bai Y., et al. "Survey of stress reactions among health care workers involved with the SARS outbreak". Psychiatric Services 55 (2004): 1055-1057.

Volume 5 Issue 6 June 2021

(C) All rights are reserved by Debashish Ghose., et al. 